J. DIFFERENTIAL GEOMETRY

45 (1997) 471-487

\title{
THE CLASS OF THE DIAGONAL IN FLAG BUNDLES
}

\author{
WILLIAM GRAHAM
}

\section{Introduction}

Let $G$ be a complex reductive group and $B$ a Borel subgroup of $G$, and let $B G$ and $B B$ denote the classifying spaces of these groups. Then $B B \rightarrow B G$ is a flag bundle: a fibration with fibers isomorphic to the flag variety $G / B$. The diagonal $\Delta \subset B B \times_{B G} B B$ can be used to define a class in $H^{*}\left(B B \times_{B G} B B\right)$. For classical groups this class has been studied by Fulton and by Pragacz and Ratajski (see [14], [15], [21], [22]). This paper studies the class of the diagonal for general $G$, from a Lie-theoretic point of view. Here $H^{*}$ denotes cohomology with complex coefficients; see Section 2 for a discussion of integer cohomology and Chow groups.

The motivation for this study comes from degeneracy loci. In its simplest form, if $V$ and $W$ are vector bundles on $M$ and $\phi: V \rightarrow$ $W$ a generic bundle map, the locus $Z \subset M$ where $\phi_{z}$ has less than maximal rank is called a degeneracy locus. More generally one can consider a vector bundle $V$ equipped with a pair of flags of subbundles $E_{1} \subset \ldots \subset E_{n}=V$ and $F_{1} \subset \ldots \subset F_{n}=V$. This corresponds to $G=G L_{n}$. For each $w \in S_{n}$ (the Weyl group of $G L_{n}$ ) there is a locus $Z_{w} \subset M$ defined by certain incidence relations between the flags $E$ and $F$. Bundles equipped with an orthogonal or symplectic form, and isotropic or Lagrangian flags of subbundles, correspond to the groups $S O(n)$ or $S p(n)$. Many schemes can be realized as degeneracy loci and for this reason general facts about such loci are useful (see [23] for a survey, and also [9], [16]).

\footnotetext{
Received September 9, 1996.
} 
The problem considered in [14], [15], [22] is to find polynomials $F_{w}$ in the Chern classes of the $E_{i}$ and $F_{j}$ such that $\left[Z_{w}\right]=F_{w} \cap[M]$. Because much is known about Chern classes, such polynomials are useful in studying the $Z_{w}$, for example, in proving that $Z_{w}$ is nonempty, or intersecting $Z_{w}$ with other subvarieties.

To solve this problem it suffices to consider the most degenerate locus, namely the diagonal $Z_{e}=\Delta$, the locus where the flags coincide, since $F_{w}$ can be obtained by applying divided difference operators to $F_{e}([14])$. As discussed below, it also suffices to consider the universal case where $M=B B \times{ }_{B G} B B$, since any other case pulls back from this. Although $B B \times{ }_{B G} B B$ is infinite-dimensional, it is a limit of compact finite dimensional manifolds, and $\Delta$ is a limit of codimension $2 d$ submanifolds (here $d$ is the complex dimension of $G / B$ ), so Poincaré duality can still be used to define a class $f \in H^{*}\left(B B \times{ }_{B G} B B\right)$. We call $f$ the class of the diagonal and write $f \cap\left[B B \times{ }_{B G} B B\right]=[\Delta]$.

Let $H \subset B$ be a maximal torus with Lie algebra $\mathfrak{h}$ and let $W$ denote the Weyl group of $H$ in $G$. Write $R=S\left(\mathfrak{h}^{*}\right), S=S\left(\mathfrak{h}^{*}\right)^{W}$. It is well-known that

$$
H^{*}(B G)=S, H^{*}(B B)=R,
$$

and it is easy to show that

$$
H^{*}\left(B B \times_{B G} B B\right)=R \otimes_{S} R .
$$

(Proposition 2.1). The class $f$ is thus an element of $R \otimes_{S} R$. The problem of finding a formula for the class of the diagonal, considered by Fulton and Pragacz-Ratajski, is essentially the problem of finding a lift $F$ of $f$ to $R \otimes \mathbb{C} R$.

The formulas of [15] are quite different from the formulas of [22]: the two constructions produce different lifts of $f$. It is difficult to compare these formulas algebraically and for this reason (and on general principles) it is desirable to have a description of $f$ which does not depend on a particular choice of lift.

The main result of this paper is the following description of $f$, which is valid for any connected reductive $G$. First note that $\operatorname{Spec} R \otimes_{S} R=$ $\mathfrak{h} \times_{\mathfrak{h} / W} \mathfrak{h}$. This scheme is a union of irreducible components $\mathfrak{h}_{w}=$ $\{(x, w x) \mid x \in \mathfrak{h}\}$. The diagonal component is $\mathfrak{h}_{1}$.

Theorem 1.1. Let $f \in R \otimes_{S} R=H^{*}\left(B B \times_{B G} B B\right)$ represent the class of the diagonal. Then $f$ satisfies and is determined by the following two properties: 
1. $f$ vanishes on each component $\mathfrak{h}_{w}$ of $\mathfrak{h} \times_{\mathfrak{h} / W} \mathfrak{h}$ with $w \neq 1$.

2. $f$ restricted to $\mathfrak{h}_{1} \cong \mathfrak{h}$ is $\prod_{\alpha>0} \alpha \in R$, where the product is over the set of positive roots.

This result is proved in Section 3 .

In Section 4 we prove a result similar to the theorem of Pragacz which was used to derive the formulas of [22]. Using this we obtain a formula (Proposition 4.2) for a lift of $f$ which is valid for any $G$. Although this formula is unwieldy, it can be combined with Theorem 1.1 to yield a result about the action of $W$ on $S\left(\mathfrak{h}^{*}\right)$ (Corollary 4.3). It would be interesting to give a purely algebraic proof of this corollary.

The remainder of this paper is primarily devoted to connections with degeneracy loci and the work of Fulton and Pragacz and Rajatski. Their work concerns a space $X$ equipped with a vector bundle $V$ and two flags $E_{1} \subset \ldots \subset E_{n}$ and $F_{1} \subset \ldots \subset F_{n}$ of subbundles of $V$. There are 4 cases corresponding to the 4 families of classical groups: $A_{n-1}$, where $V$ has rank $n ; B_{n}$ (resp. $D_{n}$ ) where $V$ is equipped with a nondegenerate quadratic form, $E_{i}$ and $F_{i}$ are isotropic subbundles, and the rank of $V$ is $2 n+1$ (resp. $2 n$ ); and $C_{n}$, where $V$ has a nondegerate skew-symmetric form, $E_{i}$ and $F_{i}$ are Lagrangian, and the rank of $V$ is $2 n$. There are also twisted versions of types $B_{n}, C_{n}$, and $D_{n}$, where the form takes values in a line bundle $L \rightarrow X$. In each case the goal is to find a formula for the locus where the flags $E$ and $F$ coincide. If $G$ is one of the classical groups $S L(n), S O(2 n+1), S O(2 n)$, or $S p(2 n)$, then $B B \times \times_{B G} B B$ is equipped with a standard vector bundle and flags as in the preceding paragraph (there is no twist by $L$ ) and the results of Fulton and Pragacz-Rajatski apply to give formulas for the class of the diagonal.

To go in the other direction, to prove formulas for degeneracy loci from the results of this paper, there are two steps. The first is to use Theorem 1.1 to prove formulas for the class of the diagonal in $B B \times{ }_{B G}$ $B B$. The second is to show that $B B \times \times_{B G} B B$ is the universal case.

The first step is carried out in Section 5, where we show that for classical groups, the formulas for the class of the diagonal $B B \times{ }_{B G} B B$ obtained from [14], [15] (by viewing the diagonal as a degeneracy locus) satisfy the conditions of Theorem 1.1. This gives a new proof that these formulas represent the class of the diagonal. I do not know of a direct algebraic proof that the formulas of [22] satisfy the conditions of Theorem 1.1; in fact comparing the methods of [22] with Theorem 1.1 is the theme of the preceding section. 
The second step is carried out in Section 6 for the case where $V$ has rank $2 n$ and a quadratic form with values in $L$ (the other cases being similar or easier). We show that given a space $X$ equipped with a vector bundle and two flags of isotropic subbundles, this data is pulled back to $X$ by a map $X \rightarrow B B \times \times_{B G} B B$. If the line bundle $L$ is trivial, then the group $G$ can be taken to be $S O(2 n)$, but for arbitrary $L$ the appropriate group is an extension of $S O(2 n)$ by $\mathbb{C}^{*}$.

Finally, note that Theorem 1.1 applies to the exceptional groups as well as to classical groups. In Section 5 we use this theorem to prove a formula for the exceptional group $G_{2}$. This formula is much more concise than the general formula of Proposition 4.2. It seems possible that with some effort one could obtain formulas of a similar character for other exceptional groups. The expression for type $G_{2}$ leads to a conjecture that certain characteristic classes, which are a priori only rational cohomology classes, are actually integral. A similar result was proved in $[10]$ for the orthogonal groups.

The results of this paper are connected with equivariant cohomology, in the following way. It is easy to see that the spaces $B B \times{ }_{B G} B B$ and $E G \times{ }^{G}(G / B)$ are isomorphic. The cohomology of the second space is by definition the $B$-equivariant cohomology $H_{B}^{*}(G / B)$. Thus, $H^{*}\left(B B \times_{B G}\right.$ $B B) \cong H_{B}^{*}(G / B)$. Under this isomorphism, the class of the diagonal corresponds to the $B$-equivariant fundamental class of a point. In [3] and [8], the problem of expressing the (Poincaré dual to) the class of a point in $G / B$ in terms of the isomorphism $H^{*}(G / B) \cong R / J$ is solved (here $J$ is the ideal in $R$ generated by positive degree elements in $S$ ). Theorem 1.1 may be therefore be viewed as a $B$-equivariant analogue of this result.

Finally, we remark that there is work of Arabia and Kostant-Kumar ([1], [2], [18], [19]) on the $T$-equivariant $K$-theory and cohomology of the flag variety (in the more general context of a Kac-Moody group). Here $T=B \cap K$ is a maximal torus in a maximal compact subgroup $K$ of $G$. Because the groups $T$ and $B$ are homotopic, we have $H_{T}^{*}(G / B) \cong$ $H_{B}^{*}(G / B)$. By means of this translation into $T$-equivariant cohomology, Theorem 1.1 follows from the work of Arabia and Kostant-Kumar; in this paper we will prove Theorem 1.1 directly. Some of the results of Kostant-Kumar are extended to equivariant Chow groups in Brion [5]; see also [13].

Acknowledgments. I would like to thank William Fulton for his helpful comments, Dan Edidin for some useful conversations, and Sam 
Evens for informing me of the work of Arabia and Kostant-Kumar.

\section{Preliminaries}

In this section we discuss a few preliminaries. Throughout this paper, we will let $H^{*}$ denote cohomology with complex coefficients. From the results with complex coefficients, results can be deduced which apply to cohomology with integral coefficients, and also to operational Chow groups. This is discussed at the end of the section.

Let $G$ denote a complex reductive group and $B$ a Borel subalgebra of $G$. The positive roots will correspond to the roots in the Lie algebra of $B$. A character $\lambda$ of $H$ extends to $B$ as usual (by sending the unipotent radical of $B$ to 1$)$. Let $M_{\lambda}$ denote the line bundle $G \times_{B} \mathbb{C}_{\lambda} \rightarrow G / B$. Then $M_{\lambda}$ corresponds to a positive divisor if the weight $-\lambda$ is dominant $[8, \S 4.6]$.

Let $E G$ denote a contractible space with a free right $G$-action. Any subgroup of $G$ acts freely on $E G$; thus, $B B=E G / B \rightarrow B G$ is a fiber bundle with fiber $G / B$. Let $L_{\lambda}$ denote the line bundle $E G \times_{B} \mathbb{C}_{\lambda} \rightarrow B B$. We have maps

$$
R=S\left(\mathfrak{h}^{*}\right) \rightarrow H^{*}(B B) \rightarrow H^{*}(G / B),
$$

where the first map takes a character $\lambda$ to $-c_{1}\left(L_{\lambda}\right)$ and the second map is restriction to a fiber. The first map is an isomorphism, and the composition induces an isomorphism $R / J \rightarrow H^{*}(G / B)$; here $J$ is the ideal in $R$ generated by the homogeneous elements of positive degree in $S=R^{W}$. Under this isomorphism the Poincaré dual of a point in $G / B$ is represented by $|W|^{-1} \prod_{\alpha>0} \alpha \bmod J([8, \S 4.5]$, [3, Theorem 3.15]; note that the map $R / J$ in both of these references takes $\lambda$ to $c_{1}\left(M_{\lambda}\right)$, which accounts for the extra factor of $(-1)^{l\left(w_{0}\right)}$, omitted in [3]).

Let $K$ be a maximal compact subgroup of $G$, and $T=B \cap K$ a maximal torus of $K$. Then as manifolds, $G / B \cong K / T$. The Weyl group $W=N_{K}(T) / T$ acts on $K / T$ on the right by $k T \cdot w=k w T$, where we use $w$ to denote both an element of $W$ and a lift to $N_{K}(T)$.

Because we want to use the $W$-action it is convenient to work with $K$ rather than $G$. Under the natural map $B K=E G / K \rightarrow B G$ (which is a homotopy equivalence), the bundle $B B \rightarrow B G$ pulls back to the bundle $B T \rightarrow B K$. Our results about the class of the diagonal in $B B \times{ }_{B G} B B$ are equivalent to results about the diagonal in $B T \times_{B K} B T$. We will therefore usually let $\Delta$ denote the diagonal in $B T \times_{B K} B T$. 
Proposition 2.1. Suppose $X \stackrel{p}{\rightarrow} Y$ is a fiber bundle with fiber $F$. Assume that $F$ has finite dimensional cohomology and suppose there exist elements $\alpha_{i}$ in $H^{*} X$ which restrict to a basis of $H^{*} F$. Then the natural map

$$
\phi: H^{*} X \otimes_{H^{*} Y} H^{*} X \rightarrow H^{*}\left(X \times_{Y} X\right)
$$

taking $x_{1} \otimes x_{2}$ to $\pi_{1}^{*}\left(x_{1}\right) \pi_{2}^{*}\left(x_{2}\right)$ is an isomorphism.

Proof. By the Leray-Hirsch theorem, the classes $\pi_{1}^{*}\left(\alpha_{i}\right)$ form a basis of $H^{*}\left(X \times_{Y} X\right)$ over $\pi_{2}^{*}\left(H^{*} X\right)$. Hence the map $\phi$ is surjective. But then both $H^{*} X \otimes_{H^{*} Y} H^{*} X$ and $H^{*}\left(X \times_{Y} X\right)$ are free $H^{*} Y$-modules of the same finite rank, so $\phi$ is injective as well. q.e.d.

Integrality. Note that $B T \times_{B K} B T \stackrel{p_{1}}{\rightarrow} B T$ is a fiber bundle with fiber $K / T$. Both $K / T$ and $B T$ have torsion-free integral cohomology which vanishes in odd dimensions. Therefore the same holds for the integral cohomology of $B T \times_{B K} B T$. Hence the class of the diagonal in $H^{*}\left(B T \times_{B K} B T ; \mathbb{Z}\right)$ is determined by the class in $H^{*}\left(B T \times_{B K} B T ; \mathbb{C}\right)$. In general we do not know which classes in $H^{*}\left(B T \times_{B K} B T ; \mathbb{C}\right)$ are integral, but the class of the diagonal is integral because it is represented by a submanifold.

Chow groups. Although in this paper we will work in cohomology, the same results are valid for operational Chow groups, in the category of schemes over $\mathbb{C}$. By work of Totaro, there is a good algebraic approximation to $B G$ : Let $V$ be a representation of $G$ and let $U \subset V$ be an open subset on which $G$ acts freely such that a quotient $U / G$ exists. If $C=V-U$ has codimension greater than $i$, then $U / G$ approximates $B G$ (in operational Chow groups) up to $\operatorname{degree} i$. Now, $U / G$ is also a topological approximation to $B G([12])$, since the homotopy groups $\pi_{j}(U / G)$ vanish for $j<2 i$. (If $\phi: S^{j} \rightarrow U$, we may view $\phi$ as a map into $V$, extend it to a map $B^{j+1} \rightarrow V$, and assume that this extended map is smooth and transversal to $C$. If $j+1<2 i+1$, transversality implies that the image of $B^{j+1}$ does not intersect $C$ and hence lies in $U$.)

Since $U / G$ is smooth, there is a cycle map

$$
A^{i}(U / G) \rightarrow H^{2 i}(U / G ; \mathbb{Z})
$$

and hence a map

$$
A^{i}(B G) \rightarrow H^{2 i}(B G ; \mathbb{Z})
$$


Now, $A^{*}(B G) \otimes \mathbb{C} \cong S\left(\mathfrak{h}^{*}\right)^{W}$, [11]. As is well-known the same is true for $H^{*}(B G)$, so (with complex coefficients) the cycle map is an isomorphism. Similarly, the map

$$
A^{i}\left(B H \times_{B G} B H\right) \rightarrow H^{2 i}\left(B H \times_{B G} B H\right)
$$

is an isomorphism (with complex coefficients). Hence with complex coefficients the formula for the diagonal in Chow cohomology agrees with that in singular cohomology.

Finally, the operational Chow groups of $B H \times_{B G} B H$ are torsion-free [11], so the remarks about cohomology apply.

\section{The main theorem}

In this section we give the proof of Theorem 1.1. First note that part (1) of the theorem, combined with the fact that the degree of $f$ is $d=$ the number of positive roots, determines $f$ up to a constant multiple. For by part (1) $f$ vanishes on $\mathfrak{h}_{1} \cap \mathfrak{h}_{w}(w \neq 1)$. Identifying $\mathfrak{h}_{1}$ with $\mathfrak{h}$, this implies $\left.f\right|_{\mathfrak{h}_{1}}$ vanishes on each root hyperplane, so $\left.f\right|_{\mathfrak{h}_{1}}$ is divisible by each positive root $\alpha$. Since the degree of $f$ is $d$, this means $\left.f\right|_{\mathfrak{h}_{1}}$ is a constant multiple of $\prod \alpha$. Part (2) then says that this constant is 1 .

To show part (1) we must show that $f\left(x, w^{-1} x\right)=0$ for all $w \neq 1$. Equivalently, we must show that $\pi_{w}(f)=0$, where $\pi_{w}: R \otimes_{S} R \rightarrow R$ is the map taking $r_{1} \otimes r_{2}$ to $r_{1} \cdot w\left(r_{2}\right)$. There is a natural right $W \times W$ action on $B T \times_{B K} B T$ given by

$$
\left(e_{1} T, e_{2} T\right) \cdot\left(w_{1}, w_{2}\right)=\left(e_{1} w_{1} T, e_{2} w_{2} T\right) .
$$

Let $i_{w}: B T \rightarrow B T \times_{B K} B T$ denote the map taking $e T$ to $(e T, e w T)$. This induces on cohomology a map $i_{w}^{*}: R \otimes{ }_{S} R \rightarrow R$. By Lemma 3.1 below, $i_{w}^{*}=\pi_{w}$.

Let $\Delta_{w}=i_{w}(B T)$. The action of $W$ on $B T$ is free, so if $w \neq 1$ then $\Delta_{w} \cap \Delta$ is empty. Since $f$ represents the class of $\Delta$, we see $i_{w}^{*}(f)=$ $\pi_{w}(f)=0$. This proves $(1)$.

Write $f_{1}=\left.f\right|_{\mathfrak{h}_{1}}$ and $\mathcal{B}=K / T$. By the remark above, $f_{1}=c \prod \alpha$, and we have only to show that $c=1$. Let $\bar{f}$ denote the image of $f_{1}$ in $R / J=H^{*}(\mathcal{B})$, so $\bar{f}=c \prod \alpha \bmod I$. Now, $\bar{f}$ is the pullback of the class of the diagonal in $\mathcal{B} \times \mathcal{B}$ by the diagonal embedding. Let $\left\{a_{w}\right\}$ and $\left\{b_{w}\right\}$ be Poincaré dual bases of $H^{*}(\mathcal{B})$, indexed by $w \in W$. The class of the diagonal in $\mathcal{B} \times \mathcal{B}$ is represented by $\sum a_{w} \otimes b_{w}$, so $\bar{f}=\sum a_{w} b_{w}$. Since $\left\{a_{w}\right\}$ and $\left\{b_{w}\right\}$ are dual bases, $a_{w} b_{w}$ represents the class of a point 
in $H^{2 d}(\mathcal{B})$. As noted above, the class of a point is $|W|^{-1} \prod \alpha \bmod J$. Thus

$$
\bar{f}=\sum a_{w} b_{w}=\sum|W|^{-1} \prod \alpha=\prod \alpha \bmod J
$$

showing that $c=1$. q.e.d.

In the proof we used the following lemma.

Lemma 3.1. The maps $i_{w}^{*}$ and $\pi_{w}$ from $R \otimes_{S} R$ to $R$ coincide.

Proof. The map $S\left(\mathfrak{t}^{*}\right) \rightarrow H^{*}(B T)$ takes a character $\lambda \in \hat{T} \subset \mathfrak{t}^{*}$ to $-c_{1}\left(L_{\lambda}\right)$, where $L_{\lambda}$ is the line bundle

$$
E K \times^{T} \mathbb{C}_{\lambda} \rightarrow E K / T=B T .
$$

On $B T \times_{B K} B T$ we have line bundles $\pi_{i}^{*}\left(L_{\lambda}\right)$, where $\pi_{i}: B T \times_{B K} B T \rightarrow$ $B T$ is the $i$-th projection. It suffices to show that

$$
i_{u}^{*} \pi_{1}^{*}\left(L_{\lambda}\right)=L_{\lambda}
$$

and

$$
i_{w}^{*} \pi_{2}^{*}\left(L_{\lambda}\right)=L_{w \lambda} .
$$

The first equation holds because $\pi_{1} \circ i_{w}$ is the identity map, and the second equation is easy to check. The result follows. q.e.d.

\section{The method of push-forwards}

Pragacz and Ratajski obtain formulas for the diagonal by applying a theorem of Pragacz ([21, Theorem 5.2]). The following result is similar to Pragacz's theorem.

Theorem 4.1. Suppose $X \stackrel{\pi}{\rightarrow} Y$ is a fibration of manifolds with fiber the compact manifold $F$. Suppose $x_{i}$ and $y_{i}(i=1, \ldots, N)$ are elements of $H^{*} X$, of pure degree, such that the sets $\left\{x_{i}\right\}$ and $\left\{y_{i}\right\}$ each restrict to a basis of $H^{*} F$. Define $b_{i j} \in H^{*} Y$ by $b_{i j}=\pi_{*}\left(x_{i} y_{j}\right)$. Then the following hold:

1. The matrix $B=\left(b_{i j}\right)$ is invertible.

2. Let $A=\left(a_{i j}\right)$ denote the matrix $\left(B^{-1}\right)^{t}$. Then the class of the diagonal $f \in H^{*}\left(X \times_{Y} X\right)$ is given by

$$
f=\sum a_{i j} x_{i} \otimes y_{j} .
$$


Proof. (1) Observe that $\pi_{*}\left(x_{i} y_{j}\right)=0$ if $\operatorname{deg} x_{i}+\operatorname{deg} y_{j}<\operatorname{dim} F$. Without loss of generality we may assume that the $x_{i}$ (resp. $y_{j}$ ) are ordered by decreasing (resp. increasing) degree. Then the matrix $B$ contains blocks along the diagonal, with each block $B_{k}$ corresponding to the ordered pairs $(i, j)$ where $\operatorname{deg} x_{i}=k$ and $\operatorname{deg} y_{j}=\operatorname{dim} F-k$. Below these blocks, the entries of $B$ are 0 . To show that $B$ is invertible it suffices to show that the block diagonal matrix $B^{\prime}=\left(b_{i j}^{\prime}\right)$ with blocks $B_{k}$ is invertible. Now, $b_{i j}^{\prime}=\int_{F} i^{*} x_{i} \cdot i^{*} y_{j}$, where $i: F \hookrightarrow X$ is the inclusion of a fiber. Thus $B^{\prime}$ is invertible by Poincaré duality applied to $F$. In more detail, let $\left\{z_{j}\right\}$ be a basis of $H^{*} F$ Poincaré dual to $\left\{i^{*} x_{k}\right\}$. Both $\left\{z_{j}\right\}$ and $\left\{i^{*} y_{k}\right\}$ are bases of $H^{*} F$, and $i^{*} y_{j}=\sum b_{i j}^{\prime} z_{i}$. On the other hand, $z_{j}=\sum r_{i j} i^{*} y_{i}$ for some $r_{i j}$. Then $\left(b_{i j}^{\prime}\right)$ and $\left(r_{i j}\right)$ must be inverse matrices.

(2) Let $\gamma$ denote the projection $X \times_{Y} X \rightarrow Y$. As in [21], if $g, h \in$ $H^{*} X$ then (with $f$ denoting the class of the diagonal)

$$
\pi_{*}(g \cdot h)=\gamma_{*}(f \cdot(g \otimes h)) .
$$

Now we can write

$$
f=\sum c_{i j} x_{i} \otimes y_{j}
$$

for some $c_{i j}$ in $H^{*} Y$. Then

$$
\begin{aligned}
b_{k l}=\pi_{*}\left(y_{l} x_{k}\right) & =\gamma_{*}\left(\left(\sum_{i, j} c_{i j} x_{i} \otimes y_{j}\right) \cdot\left(y_{l} \otimes x_{k}\right)\right) \\
& =\sum_{i, j} c_{i j} \pi_{*}\left(x_{i} y_{l}\right) \pi_{*}\left(x_{k} y_{j}\right) \\
& =\sum_{i, j} c_{i j} b_{i l} b_{k j} \\
& =\left(B C^{t} B\right)_{k l} .
\end{aligned}
$$

That is, $B=B C^{t} B$, so $C=\left(B^{-1}\right)^{t}=A$. q.e.d.

In the situation of the theorem, write $A=H^{*} X, B=H^{*} Y$. Then $A$ is a free $B$-module of finite rank. Think of $B$ as the ground ring, and define an inner product $():, A \otimes_{B} A \rightarrow B$ by $\left(a_{1}, a_{2}\right)=\pi_{*}\left(a_{1} a_{2}\right)$. Using this inner product we can identify $A$ with $A^{*}=\operatorname{Hom}_{B}(A, B)$. Thus $A \otimes_{B} A \cong A^{*} \otimes_{B} A \cong \operatorname{Hom}_{B}(A, A)$, where $a_{1} \otimes a_{2}$, viewed as a map from $A$ to $A$, is given by

$$
\left(a_{1} \otimes a_{2}\right)(a)=\left(a_{1}, a\right) a_{2}
$$


The preceding result implies that the class of the diagonal, viewed as a map from $A$ to $A$, is the identity.

Now let $X=B T, Y=B K, H^{*} X=R, H^{*} Y=S$. Since we can view $Y$ as a limit of compact manifolds, the above theorem still holds. The map $\pi_{*}: R \rightarrow S$ is given by

$$
\pi_{*}(r)=\frac{\sum(-1)^{w} w(r)}{\prod_{\alpha>0} \alpha}=\partial_{w_{0}}(r),
$$

where $\partial_{w_{0}}$ is the divided difference operator corresponding to the long element $w_{0}$ of $W$. The first equality is from [4] and the second from [7]. Hence the inner product coincides in type $A$ with the inner product introduced by Lascoux-Schutzenberger [20]. Just as in type $A$ we have $\left(\partial_{\alpha} r_{1}, r_{2}\right)=\left(r_{1}, \partial_{\alpha} r_{2}\right)$.

The above results imply the following explicit formula for the class the diagonal (i.e., for a lift of $f$ ). First note that we can obtain an explicit basis of $R$ as $S$-module; one choice is to let $p_{e}$ be any element of $R$ which agrees with $|W|^{-1} \prod_{\alpha>0} \alpha \bmod J$, and then set $p_{w}=\partial_{w^{-1}} p_{e}$. If we choose two such bases we obtain the following proposition.

Proposition 4.2. Let $\left\{p_{w}\right\}$ and $\left\{q_{w}\right\}$ be bases of $R$ over $S$, indexed by $w \in W$; assume that $p_{w}$ and $q_{w}$ have pure degree. Let $b_{u v}=\partial_{w_{0}}\left(p_{u} q_{v}\right)$ and let $\left(a_{u v}\right)$ be the inverse transpose matrix to $\left(b_{u v}\right)$. Then the class of the diagonal is represented by

$$
\sum_{u, v \in W} a_{u v} p_{u} \otimes q_{v}
$$

q.e.d.

This formula is explicit but complicated. Other formulas for lifts of $f$ to $R \otimes \mathbb{C} R$ are discussed in the next section.

Let $m: R \otimes_{S} R \rightarrow R$ denote the multiplication map. Identifying $R \otimes{ }_{S} R$ with $\operatorname{Hom}_{S}(R, R)$, we will view $m$ as a map from $\operatorname{Hom}_{S}(R, R)$ to $R$. Then the inner product $\pi_{*} \circ m$ is just the trace map $\operatorname{Hom}_{S}(R, R) \rightarrow$ $S$. As an $S$-module, $R$ is free of rank $|W|$, and the natural representation of $W$ on $R$ over $S$ is equivalent to the regular representation (see [6]). Hence

$$
\pi_{*} \circ m(1)=\operatorname{Trace}(1)=|W|,
$$

and if $w \in W$ is not equal to 1 , then

$$
\pi_{*} \circ m(w)=\operatorname{Trace}(w)=0 .
$$


From the geometric arguments of this paper we see that the following stronger statement is true.

Corollary 4.3. Let $w \in W$ and view $w$ as an element of

$$
\operatorname{Hom}_{S}(R, R) \cong R \otimes_{S} R \text {. }
$$

If $w \neq 1$ then

$$
m(w)=0,
$$

and

$$
m(1)=\prod_{\alpha>0} \alpha
$$

Proof. The identity map in $\operatorname{Hom}_{S}(R, R)$ corresponds to $f \in R \otimes S R$, and the element $w \in \operatorname{Hom}_{S}(R, R)$ corresponds to $(1 \times w) f$. By Theorem 1.1 , we have $m(f)=\prod_{\alpha>0} \alpha$ and $m((1 \times w) f)=0$ for $w \neq 1$. q.e.d.

It would be interesting to have a purely algebraic proof of this result which applies to root systems of type $H_{3}$ and $H_{4}$ (which are not covered by the corollary).

\section{Expressions for the class of the diagonal}

In this section we discuss some expressions for the class of the diagonal. For the classical groups, expressions follow from the work of Fulton, and of Pragacz and Ratajski for the orthogonal and symplectic groups. We will sketch a verification that Fulton's expressions satisfy the conditions of Theorem 1.1, thus providing another proof that they represent the class of the diagonal. We will also give an expression for the exceptional group $G_{2}$. In this section it is not necessary to interpret the terms of these formulas as Chern classes of subbundles (but see Section 6); we have only to check the conditions of Theorem 1.1.

We remark that the method of proof in [15] is to construct a sequence $M=Z_{0} \supset Z_{1} \supset \cdots \supset Z_{k}=\Delta$, where $Z_{i+1}$ is the zero-locus of a vector bundle on $Z_{i}$. This yields a formula for the class of the diagonal. Algebraic arguments convert this into a formula with a more "determinantal" appearance. It turns out that this algebra is precisely what is needed to verify that these formulas satisfy the conditions of Theorem 1.1 , even though the proof of this theorem is quite different from the proofs of $[15]$. 
For the classical groups we have standard realizations of $\mathfrak{h}^{*}$ and of the roots. For $B_{n}, C_{n}, D_{n}$ we will write

$$
S\left(\mathfrak{h}^{*}\right) \otimes \mathbb{C} S\left(\mathfrak{h}^{*}\right)=\mathbb{C}\left[x_{1}, \ldots, x_{n}, y_{1}, \ldots, y_{n}\right] ;
$$

for $A_{n-1}$ we impose the relations $\sum x_{i}=\sum y_{i}=0$. Our convention is that $x_{i}$ and $y_{i}$ represent the same coordinate on $\mathfrak{h}$; on $\mathfrak{h} \times \mathfrak{h}, x_{i}$ corresponds to the first factor and $y_{i}$ to the second. (We note this because under the convention of [15] our $y_{i}$ would be $y_{n+1-i}$.) For $A_{n-1}$, the expression of $[14]$ is

$$
F(x, y)=\prod_{i<j}\left(x_{i}-y_{j}\right) .
$$

Since the Weyl group acts by permuting the coordinates, and the positive roots are $x_{i}-x_{j}(i<j)$, the conditions of Theorem 1.1 are clear. For $D_{n}$, the expression of [15] is

$$
F(x, y)=\prod_{i<j}\left(x_{i}-y_{j}\right) \cdot F_{1}(x, y) .
$$

Here $F_{1}(x, y)=\operatorname{det}\left(c_{i j}\right)$, where $\left(c_{i j}\right)$ is the $n \times n$ matrix whose $(i, j)$ entry is

$$
c_{i j}=\frac{1}{2}\left(e_{k}(x)+e_{k}(y)\right), k=n+1+j-2 i .
$$

Here $e_{k}(x)$ and $e_{k}(y)$ denote the $k$-th elementary symmetric function in the variables $x_{i}$ and $y_{i}$, respectively. The only properties of $F_{1}(x, y)$ we will use are that it is invariant under permutations of the $x_{i}$ or $y_{i}$, and that if $\epsilon_{i}= \pm 1$ (with an even number of $\epsilon_{i}$ equal to -1 ), then

$$
F_{1}\left(x_{1}, \ldots, x_{n}, \epsilon_{1} x_{1}, \ldots, \epsilon_{n} x_{n}\right)
$$

is 0 unless all $\epsilon_{i}$ are equal to 1 , in which case it equals $\prod_{i<j}\left(x_{i}+x_{j}\right){ }^{1}$ The Weyl group of $D_{n}$ consists of signed permutations on $n$ letters, which involve only an even number of sign changes. If $w$ is in the Weyl group, then $F(x, w x)$ is 0 unless all the signs are 1; in that case $w$ is just a permutation, and $F(x, w x)$ clearly vanishes unless $w$ is the identity permutation. Likewise,

$$
F(x, x)=\prod_{i<j}\left(x_{i}^{2}-x_{j}^{2}\right)=\prod_{\alpha>0} \alpha(x) .
$$

\footnotetext{
there.

${ }^{1}$ The analogous result for type $C_{n}$ is proved in detail in [15]; this is indicated
} 
This verifies Fulton's formulas for types $A$ and $D$; types $B$ and $C$ are checked similarly.

For type $G_{2}$, we use the description of [17]: we realize the maximal torus of $G_{2}$ as the subset of $\mathbb{C}^{3}$ where the coordinates add up to 0 , and write

$$
S\left(\mathfrak{h}^{*}\right) \otimes \mathbb{C} S\left(\mathfrak{h}^{*}\right)=\mathbb{C}\left[x_{1}, x_{2}, x_{3}, y_{1}, y_{2}, y_{3}\right]
$$

modulo the relations $\sum x_{i}=\sum y_{i}=0$. The 6 positive roots are $x_{i}-x_{j}$ $(i<j), x_{i}+x_{j}-2 x_{k}$. Note that as a function on $\mathfrak{h}, x_{i}+x_{j}-2 x_{k}=-3 x_{k}$. The Weyl group is generated by permutations of the coordinates and the map multiplication by -1 . It is easy to check that

$$
-\frac{27}{2}\left(x_{1}-y_{2}\right)\left(x_{1}-y_{3}\right)\left(x_{2}-y_{3}\right)\left(x_{1} x_{2} x_{3}+y_{1} y_{2} y_{3}\right)
$$

satisfies the conditions of Theorem 1.1 and hence represents the class of the diagonal. This suggests the conjecture that $\frac{1}{2}\left(x_{1} x_{2} x_{3}+y_{1} y_{2} y_{3}\right)$ is an integral cohomology class. This is like [10, Theorem 1] (which concerned the orthogonal groups), and probably can be proved similarly.

\section{Loci defined by flags of vector bundles}

The papers of Fulton and Pragacz-Ratajski give formulas for degeneracy loci defined by flags of vector bundles. As indicated in the introduction, the spaces $B B \times \times_{B G} B B$ are the universal cases, in that formulas derived for this space can be pulled back to an arbitrary space. In this section we explain this in detail. For simplicity, we will only consider the even orthogonal case and its twisted analogue; there are similar (simpler) versions for the other classical groups. At the end of the section we indicate the modifications to the argument necessary to apply it to Chow groups.

Suppose that $V \rightarrow X$ is a rank $2 n$ vector bundle equipped with a nondegenerate quadratic form with values in a line bundle $L \rightarrow X$. Suppose that $E_{1} \subset \ldots \subset E_{n}=V$ and $F_{1} \subset \ldots \subset F_{n}=V$ are maximal flags of isotropic subbundles, so $E_{i}$ and $F_{i}$ are isotropic of rank $i$. We will assume that $E$ and $F$ are in the same family, i.e., $\operatorname{dim}\left(E_{n}\right)_{x}=$ $\operatorname{dim}\left(F_{n}\right)_{x}$ for all $x \in X$. (The case of opposite families can be reduced to this.) Formulas for the locus where the flags $E$ and $F$ coincide are given in [15] and (if $L$ is trivial) in [22]. In this section we will show that the formulas of [15] can be deduced by pulling back to $X$ from $B B \times \times_{B G} B B$ for a suitable choice of $G$. In the untwisted case where $L$ 
is trivial we can take $G=S O(2 n)$. As noted in [15], while a formula for the general (twisted) case can be obtained from the untwisted case, information about 2-torsion is lost. It is better to deal directly with the twisted case.

For the twisted case, the appropriate group $G$ is an extension of $S O(2 n)$ by $\mathbb{C}^{*}$, defined as follows. Let $V^{\prime}=\mathbb{C}^{2 n}$ with the quadratic form defined by $\left(e^{i}, e^{2 n+1-j}\right)^{\prime}=\delta_{i j}$. Let $G^{\prime}$ denote the set of $g \in G L(2 n)$ such that for all $v, w \in V^{\prime},(g v, g w)^{\prime}=\gamma(g)(v, w)^{\prime}$. Here $\gamma(g)$ is a constant depending only on $g$, and the map $\gamma: G \rightarrow \mathbb{C}^{*}$ is a homomorphism. The condition $(g v, g w)^{\prime}=\gamma(g)(v, w)^{\prime}$ implies that $\operatorname{det}(g)^{2}=\gamma(g)^{2 n}$. The group $G^{\prime}$ has 2 components: the identity component, defined by the condition $\operatorname{det}(g)=\gamma(g)^{n}$, and the other component defined by $\operatorname{det}(g)=$ $-\gamma(g)^{n}$. Let $G$ denote the identity component of $G^{\prime}$.

The group $G$ has $V^{\prime}$ as standard representation; it also has a 1dimensional representation (denoted by $L^{\prime}$ ) defined by the character $\gamma$. Over $B G$ we have the vector bundle $\mathcal{V}=E G \times{ }^{G} V^{\prime}$ and the line bundle $\mathcal{L}=E G \times^{G} L^{\prime}$. A section $v$ of $\mathcal{V}$ is a map $E G \rightarrow V^{\prime}$ satisfying $v(e \cdot g)=g^{-1} v(e)$. There is a similar description of sections of $\mathcal{L}$. The vector bundle $\mathcal{V}$ has a quadratic form with values in $\mathcal{L}$ : if $v_{1}$ and $v_{2}$ are two sections of $\mathcal{V}$, then $\left(v_{1}, v_{2}\right)$ is the section of $\mathcal{L}$ defined by $\left(v_{1}, v_{2}\right)(e)=\left(v_{1}(e), v_{2}(e)\right)^{\prime}$.

Let $E_{1}^{\prime} \subset \ldots \subset E_{n}^{\prime}$ be the flag of isotropic subspaces of $V^{\prime}$ with $E_{i}^{\prime}$ equal to the span of $e^{1}, \ldots, e^{i}$. Let $B \subset G$ denote the stabilizer of the flag $E^{\prime}$. The pullback of $\mathcal{V}$ to $B B$ has a flag $\mathcal{E}$ of isotropic subbundles defined by $\mathcal{E}_{i}=E G \times{ }^{B} E_{i}^{\prime}$. The pullback of $\mathcal{V}$ to $B B \times{ }_{B G} B B$ has two isotropic flags. We will abuse notation and write $\mathcal{E}=\pi_{1}^{*} \mathcal{E}, \mathcal{F}=\pi_{2}^{*} \mathcal{E}$.

Suppose $V \rightarrow X$ has a quadratic form with values in a line bundle $L$. We show that there is a classifying map from $X$ to $B G$, as follows. Cover $X$ by open sets $U_{i}$ on which $V$ and $L$ are trivial. Let $s_{i}$ denote the trivializing section of $L$ on $U_{i}$, and assume our trivializing sections $v_{i}^{1}, \ldots, v_{i}^{2 n}$ of $\left.V\right|_{U_{i}}$ satisfy $\left(v_{i}^{k}, v_{i}^{2 n+1-j}\right)=\delta_{k j} s_{i}$. These trivializing sections define, in the obvious way, maps $\phi_{i}: U_{i} \times V^{\prime} \rightarrow V_{U_{i}}$ and $g_{j i}=\phi_{j}^{-1} \circ \phi_{i}: U_{j i} \times V^{\prime} \rightarrow U_{j i} \times V^{\prime}$ (here $U_{j i}=U_{j} \cap U_{i}$ ). As usual, we may view $g_{j i}$ as a map from $U_{j i}$ into $G L\left(V^{\prime}\right)$. By construction, if $x \in U_{j i}$ and $v, w \in V^{\prime}$, then $\left(g_{j i}(x) v, g_{j i}(x) w\right)^{\prime}=\frac{s_{i}}{s_{j}}(x)(v, w)^{\prime}$. Thus, our transition functions $g_{j i}$ take values in the group $G^{\prime}$. Hence they define a $G^{\prime}$-principal bundle $P \rightarrow X$ with associated bundle $P \times{ }^{G^{\prime}} V^{\prime}=V$. This bundle is pulled back from the universal bundle by a classifying map $\chi: X \rightarrow B G^{\prime}$. By construction, $V=\chi^{*} \mathcal{V}, L=\chi^{*} \mathcal{L}$, and the quadratic 
form on $V$ is the pullback of the form on $\mathcal{V}$.

If $V$ is equipped with a flag of isotropic subbundles $E_{1} \subset \cdots \subset E_{n}$, then we can assume our trivializations of $\left.V\right|_{U_{i}}$ have the additional property that $v_{i}^{j}$ is a basis for $\left.\left(E_{j} / E_{j-1}\right)\right|_{U_{i}}$. Thus our transition functions $g_{j i}$ will take values in $B \subset G$. Hence $\chi$ lifts to a classifying map $\tilde{\chi}: X \rightarrow B B$, and the pullback of $\mathcal{E}_{i}$ is $E_{i}$. Likewise if $X$ has two isotropic flags $E$ and $F$, then this data is all pulled back to $X$ by a map $X \rightarrow B B \times{ }_{B G} B B$, and the locus where $E$ and $F$ coincide is the pullback of the diagonal in $B B \times{ }_{B G} B B$.

This shows that $B B \times_{B G} B B$ is indeed the universal case for this problem. We now wish to find a formula for the diagonal in terms of the Chern classes of $\mathcal{E}, \mathcal{F}$, and $\mathcal{L}$. The Lie algebra $\mathfrak{g}$ of $G$ is $\mathfrak{g}=\mathfrak{g}_{1} \oplus \mathfrak{z}$, where $\mathfrak{g}_{1}=\mathfrak{s o}(2 n)$ and $\mathfrak{z}=\{c I \mid c \in \mathbb{C}\}$. A maximal torus of $\mathfrak{g}$ is $\mathfrak{h}=\mathfrak{h}_{1} \oplus \mathfrak{z}$, where $\mathfrak{h}_{1}$ is the diagonal maximal torus of $\mathfrak{s o}(2 n)$. This splitting gives a dual splitting $\mathfrak{h}^{*}=\mathfrak{h}_{1}^{*} \oplus \mathfrak{\mathfrak { j }}^{*}$. Define elements $x_{i}, z \in \mathfrak{h}^{*}$ as follows. Any $A \in \mathfrak{h}$ can be written as $A=A_{1}+c I$, where $A_{1} \in \mathfrak{h}_{1}$ is a diagonal matrix with entries $\left(a_{1}, \ldots, a_{n},-a_{n}, \ldots,-a_{1}\right)$. Set $x_{i}\left(A_{1}+c I\right)=a_{i}$, $z\left(A_{1}+c I\right)=c$. Thus, $x_{1}, \ldots, x_{n}, z$ form a basis for $\mathfrak{h}^{*}$, and $x_{1}, \ldots, x_{n}$ is the standard basis for $\mathfrak{h}_{1}^{*}$. Write

$$
S\left(\mathfrak{h}^{*}\right) \otimes_{\mathbb{C}} S\left(\mathfrak{h}^{*}\right)=\mathbb{C}\left[x_{1}, \ldots, x_{n}, z, y_{1}, \ldots, y_{n}, w\right],
$$

where $x_{i}$ (resp. $y_{i}$ ) are the standard basis of the first (resp. second) copy of $\mathfrak{h}_{1}^{*}$, and $z$ (resp. $w$ ) is the element above in the first (resp. second) copy of $\mathfrak{z}^{*}$.

Because $\mathfrak{g}$ is the direct sum of $\mathfrak{s o}(2 n)$ and an abelian Lie algebra, the roots and Weyl group for $\mathfrak{g}$ are the same as for $\mathfrak{s o}(2 n)$. It follows that the class of the diagonal is given by the same formula as for $G=S O(2 n)$ (equation (5.1)). Note that this expression does not involve $z$ and $w$.

Finally, we must relate the $x_{i}$ to Chern classes of the bundles $\mathcal{E}_{i}$ on $B B$. The restriction of the character $\gamma$ of $G$ to $H$ is $2 z$. Now, $\mathcal{E}_{i} / \mathcal{E}_{i-1}$ is the line bundle $E G \times{ }^{B}\left(E_{i}^{\prime} / E_{i-1}^{\prime}\right)$. The Lie algebra $\mathfrak{h}$ acts on $E_{i}^{\prime} / E_{i-1}^{\prime}$ by the character $x_{i}+z$. (Note that $x_{i}$ and $z$ define characters of $\mathfrak{h}$ but not $H$. However, both $x_{i}+z$ and $2 z$ integrate to characters of $H$.) In the notation of Section $2, \mathcal{E}_{i} / \mathcal{E}_{i-1}$ is the line bundle $L_{x_{i}+z}$. Hence under the isomorphism $S\left(\mathfrak{h}^{*}\right)=H^{*}(B B)$ defined in Section 2, we have $-\left(x_{i}+z\right)=c_{1}\left(\mathcal{E}_{i} / \mathcal{E}_{i-1}\right)$. Also, the pullback of $\mathcal{L}$ to $B B$ (which we will still denote by $\mathcal{L}$ ) is the line bundle $L_{2 z}$, so under our isomorphism, $z=-\frac{1}{2} c_{1}(\mathcal{L})$. Thus $x_{i}=-c_{1}\left(\mathcal{E}_{i} / \mathcal{E}_{i-1}\right)+\frac{1}{2} c_{1}(\mathcal{L})$.

This calculation is for bundles on $B B$. Recall that on $B B \times_{B G} B B$ we have bundles $\mathcal{E}$ and $\mathcal{F}$ (we abuse notation and write $x_{i}=\pi_{1}^{*} x_{i}$, 
$\left.y_{i}=\pi_{2}^{*} x_{i}, \mathcal{E}=\pi_{1}^{*} \mathcal{E}, \mathcal{F}=\pi_{2}^{*} \mathcal{E}\right)$. The preceding calculation implies that for these bundles on $B B \times{ }_{B G} B B$ we have $x_{i}=-c_{1}\left(\mathcal{E}_{i} / \mathcal{E}_{i-1}\right)+\frac{1}{2} c_{1}(\mathcal{L})$, $y_{i}=-c_{1}\left(\mathcal{F}_{i} / \mathcal{F}_{i-1}\right)+\frac{1}{2} c_{1}(\mathcal{L})$. If we substitute these expressions into the formula (5.1) we recover the formula of [15].

The preceding arguments are for cohomology, but if $X$ is a scheme over $\mathbb{C}$, they work for Chow groups with only minor modification. This argument uses some results of Totaro on his algebraic model for $B G$, which are explained in [11]. The only difference is in the part of the argument showing that $B B \times \times_{B G} B B$ is universal. The vector bundle $V$ on $X$ is still associated to a principal $G$-bundle $P \rightarrow X$. The presence of flags of isotropic subbundles implies that this bundle is locally trivial in the Zariski topology [10]. In the algebraic setting we cannot assume that $P \rightarrow X$ is pulled back from the algebraic model for $B G$. However, if $X$ is quasi-projective, we can find an affine bundle $\pi: X^{\prime} \rightarrow X$ such that $P^{\prime}=\pi^{*} P$ is pulled back from an algebraic model for $B G$. The formula for the locus where the (pulled-back) flags coincide holds on $X^{\prime}$ by the arguments above. Since $\pi^{*}$ is an isomorphism of Chow groups, the formula holds on $X$. If $X$ is not quasi-projective, then consider a Chow envelope $X^{\prime} \rightarrow X$. The pullback is injective on Chow groups, so it suffices to prove the formula on $X^{\prime}$, but it holds there because by definition $X^{\prime}$ is quasi-projective.

\section{References}

[1] A. Arabia, Cohomologie T-équivariant de $G / B$ pour a groupe $G$ de Kac-Moody, C.R. Acad. Sci. Paris, Sér. 1, 302 (1986) 631-634.

[2] Cycles de Schubert et cohomologie équivariant de $K / T$, Invent. Math. 85 (1986) 39-52.

[3] I. M. Bernstein, I. M. Gelfand \& S. I. Gelfand, Schubert cells and cohomology of the spaces $G / P$, Russian Math. Surveys 28 (1973) 1-26.

[4] M. Brion, The push-forward and Todd class for flag bundles, Parameter Spaces, Banach Center Publications 36 (1996).

[5] _ Equivariant Chow groups for torus actions, Preprint, 1996.

[6] C. Chevalley, Invariants of finite groups generated by reflections, Amer. J. Math 77 (1955) 778-782.

[7] M. Demazure, Invariants symétriques entiers des groupes de Weyl et torsion, Invent. Math. 21 (1973) 287-301. 
[8] Désingularisation des variétés de Schubert généralisées, Ann. Sci. École Norm. Sup. 7 (1974) 53-88.

[9] C. De Concini \& P. Pragacz, On the class of Brill-Noether for Prym varieties, Math. Ann. 302 (1995) 687-697.

[10] D. Edidin \& W. Graham, Characteristic classes and quadric bundles, Duke Math. J. 78 (1995) 277-299.

[11] Characteristic classes in the Chow ring, J. Algebraic Geom., to appear.

[12] - Equivariant intersection theory, Invent. Math., to appear.

[13] L L L Lalization in equivariant intersection theory and the Bott residue formula, Preprint.

[14] W. Fulton, Flags, Schubert polynomials, degeneracy loci and determinantal formulas, Duke Math. J. 65 (1992) 381-420.

[15] Schubert varieties in flag bundles for the classical groups, Proc. Conf. in Honor of Hirzebruch's 65th Birthday, Bar Ilan, Israel Math. Conf. Proc. 9 (1995) Amer. Math. Soc.

[16] _ Determinantal formulas for orthogonal and symplectic degeneracy loci, J. Differential Geom. 43 (1996) 276-290.

[17] J. E. Humphreys, Introduction to Lie algebras and representation theory, Springer, Berlin, 1972.

[18] B. Kostant \& S. Kumar, The nil Hecke ring and cohomology of G/P for a KacMoody group, Adv. Math. 62 (1986) 187-237.

[19],$T$-equivariant $K$-theory of generalized flag varieties, J. Differential Geom. 32 (1990) 549-603.

[20] I. G. Macdonald, Notes on schubert polynomials, Publ. Labor. Combin. Inform. Math., Univ. du Quebec à Montréal, 1991.

[21] P. Pragacz, Symmetric polynomials and divided differences in formulas of intersection theory, Parameter Spaces, Banach Center Publ., Vol. 36, 1996.

[22] P. Pragacz \& J. Ratajski, Formulas for Lagrangian and orthogonal degeneracy loci: the $\tilde{Q}$-polynomials approach, Compositio Math., to appear.

[23] L. Tu, Degeneracy loci, Proc. Conf. Algebraic Geom. (Berlin 1985), Teubner, Leipzig, 1986, 296-305.

Institute for Advanced Study 